\title{
Low power consumption receiver on silicon
}

\author{
L. Virot ${ }^{1,2,3}$, D. Marris-Morini ${ }^{1}$, D. Benedikovic ${ }^{1}$, C. Alonso-Ramos ${ }^{1}$, \\ J-M. Hartmann ${ }^{2}$, E. Cassan ${ }^{1}$, P. Crozat ${ }^{1}$, X. Le Roux ${ }^{1}$, C. Baudot ${ }^{3}$, F. Boeuf ${ }^{3}$, \\ J-M. Fédéli ${ }^{2}$, L. Vivien ${ }^{1 *}$ \\ ${ }^{I}$ Institut d'Electronique Fondamentale, Univ. Paris-Sud, CNRS UMR 8622, Univ. Paris Saclay, Bât. 220,91405 \\ Orsay, France; \\ ${ }^{2}$ ST Microelectronics, 850 rue Jean Monnet 38920 Crolles, France \\ ${ }^{3}$ CEA, LETI, Minatec Campus 17 rue des Martyrs, 38054 Grenoble cedex 9 France \\ "laurent.vivien@u-psud.fr
}

Silicon is the mainstream material in the electronic industry and it is rapidly expanding its dominance into the field of photonics. Indeed, silicon photonics has been the subject of intense research activities to pave the way for next generation of energy-efficient high-speed computing, information processing and communications systems. The trend is to use optics in intimate proximity to the electronic circuit, which implies a high level of optoelectronic integration. Over the last decade, the field of silicon photonics has advanced at a remarkable pace. Recent advances in integrated germanium receiver will be presented including the developments of a new Ge-based detector integration and avalanche Ge structures. 